

\title{
VSAT-Based Satellite Wide Area Networks ${ }^{1}$ \\ Jin-Fu Chang ${ }^{2}$ and Sung-Hui Lin \\ Electrical Engineering Department \\ National Taiwan University \\ Taipei, Taiwan 10764
}

\begin{abstract}
This paper evaluates the performance of VSAT-based satellite wide area networks. Very small aperture terminals are used as gateways to interconnect local area networks through satellite to form a wide area network. This paper concentrates on analyzing the delay of the network which is the time needed to send a packet from a station in the source LAN to a station in the destination LAN. Several access protocols are considered for comparison. It is observed through numerical examples that TDMA on the uplink and TDM broadcast on the downlink offers the best delay performance.
\end{abstract}

\section{Introduction}

The appearance of very small aperture terminal(VSAT) systems marks a turning point in satellite industry. VSAT is attractive in its small size and low cost and has great potential in many applications. It can be used in applications such as inventory management, electronic fund transfer, file transfer, $\mathrm{e}-$ mail, and etc. in wholesale, finance, information, and service industry.

Sketched in Fig. 1 is the basic architecture of a VSAT network. One application of such network is data retrieved from the database installed in the hub station. In such application, the satellite is used as a relay for a VSAT to access the data stored in the hub station. Tutorial papers on various aspects of VSAT systems can be found in [1]-[7]. [8]-[9] are two recent articles to discuss some technical issues in VSAT sytems.

The idea of using VSAT systems as gateways to interconnect different local area networks(LANs) to form a satellite wide area network(SWAN) was first mentioned in [2] as one of the future applications of VSAT. Depicted in Fig. 2 is the architecture of the system postulated in 22]. The purpose of this paper is to explore the subject further and analyze the delay performance of such VSAT-based satellite wide area network.

The system is studied under the following assumptions.

A.1. All LANs are identical

1. This work is supported jointly by the National Science Council and the Telecommunication Labs of the Ministry of Communications through Grants NSC-80-0404-E002-16 and TL-NSC-80-2101.

2. Currently with National Central University, Chung-Li, Taiwan 320.

Also with Institute of Information Science, Academia Sinica, Tajpei, Taiwan. 
A.2. Each LAN is a ring or bus type network. We shall not in the following try to distinguish between ring and bus. Instead we shall only use the word ring in the sequel.

A.3. Each LAN consists of rings in dual. The dual structure is adopted mainly for fault tolerence. However to better utilize the network resource we shall use the inner and outer ring for different purposes. One of them is used to send packet from one station to another station in the LAN or to the satellite via the VSAT gateway. The other is used to receive packets from the satellite via the VSAT gateway and then forward them to appropriate destination stations in the LAN. We even assume that these two rings are allowed to have different capacities.

A.4. Each VSAT gateway is equipped with two sufficiently large queues: the outbound and inbound queues. The outbound queue is used to buffer packets outgoing to a different LAN while the inbound queue is used to buffer packets received form the satellite.

A.5. Both the uplink channel to and downlink channel from satellite have the same transmission capacity. The channel time is slotted in such a way that each slot is good for the transmission of a packet.

A.6. The operation of each ring is constructed on the circulation of a token. A station has to first seize a token in order to transmit packets. In this paper we further assume that each station can send at most one packet each time a token is captured.

Clearly, if a station wishes to send a packet to some station in another LAN. The packet has to be first sent from the source destination to the VSAT gateway, next to the satellite via the uplink channel, then to the destination VSAT gateway via the downlink channel, and finally to the destination station. The purpose of this paper is to evaluate the end-to-end delay in the above transmission. In calculating the delay from VSAT gateway to satellite we need to specify the protocol used on the uplink channel. Similarly we also need to specify the protocol used on the downlink channel. In this paper three combinations of uplink/downlink protocols will be considered for comparison. They are FDMA/FDM, FDMA/TDM and TDMA/TDM.

\section{Numerical examples and discussions}

Extensive numerical calculations for each of the above three protocols based on the results derived in [11]. These calculations have also been verified by computer simulations to support the validity of our mathematical derivations. Due to the restriction on the length of a submitted paper we shall present only the comparison among these three protocols.In Fig. 3 we observe that TDMA/TDM generally gives the best performance. Therefore our recommendation is to use TDMA/TDM to link local area networks through VSATs.

Although in the present paper we concentrate our study on uniform network, these protocols can be very easily modified to adapt to variation in load distribution. For example busier VSATs can be given more bandwidth in FDMA or more time slots in TDMA. However the analysis should be of no doubt become much more involved. In [11] we also consider an alternative method in dealing with nonuniform traffic called random-select (RS)/TDM. In RS, all the VSATs are equipped with an identical random number generator running from the same starting point or seed. The right of transmission is governed by the random number generator and busier VSATs can be given more right to send.

In this paper, each LAN contains dual loops. The performance of single-loop LANs remains to be studied. But the work is expected to be much more tedious. Besides, LAN architectures other than token ring can also be considered for comparison. 


\section{References}

[1] D. Chakraborty, "VSAT Communications networks-An overview," IEEE Communications Magazine, Vol. 26, pp. 10-24, May 1988.

[2] K. M. S. Murthy and K. G. Gordon, "VSAT networking concepts and new applications development," IEEE Communications Magazine, Vol. 27, pp. 43-49, May 1989.

[3] J. Stratigos and R. Mahindru, "Packet switch architectures and user protocol interfaces for VSAT networks," IEEE Communications Magazine, Vol. 26, pp. 39-47, July 1988 .

[4] D. M. Chitre and J. S. McCoskey, "VSAT networks: architecture, protocols, and management," IEEE Communication Magazine, Vol. 26, pp. 28-38, July 1988.

[5] F. M. Naderi and W. W. Wu, "Advanced satellite concepts for future . generation VSAT networks," IEEE Communication Magazine, pp. 13-22, Sept. 1988.

[6] K. M. S. Murthy, et al., "VSAT network examples," IEEE Communications Magazine, Vol. 27, pp. 50-57, May 1989.

[7] L. S. Golding, et al., "VSATs: expert views on future trends," IEEE Communications Magazine, Vol. 27, pp. 58-64, May 1989.

[8] T. T. Hu, "Personal Communications via VSAT networks," IEEE J. Selected Areas in Communications, SAC-7, pp. 235-245, Feb. 1989

[9] L. S. Liang and J. F. Chang, "Response time calculation for VSAT networks," IEEE Milcom 90, 59.5, Monterey, CA, September 1990.

[10] P. J. Kuehn, "Multi-queue systems with non-exhaustive cyclic service," The Bell System Tech. J., 58, pp. 678-698, 1978.

[11] Sung-Hui Lin, "VSAT-Based Satellite Area Networks," MS Thesis, Department of Electrical Engineering, National Taiwan Unviersity, Taipei, Taiwan, June 1991. 


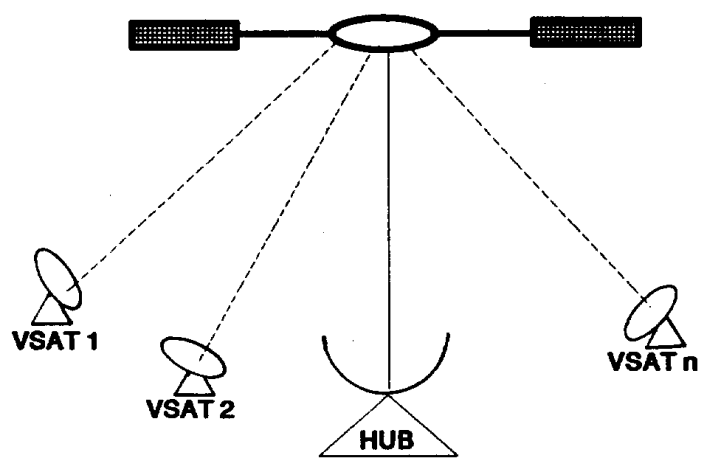

Fig. 1 Basic architecture of VSAT network

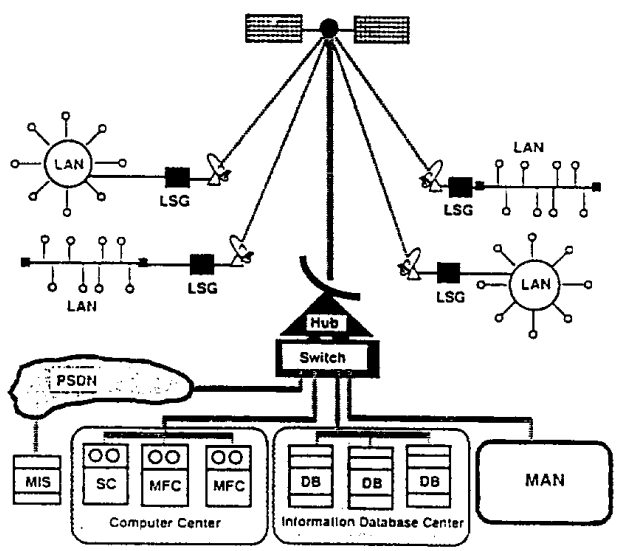

Fig. 2 The architecture of SWAN proposed in [2] 


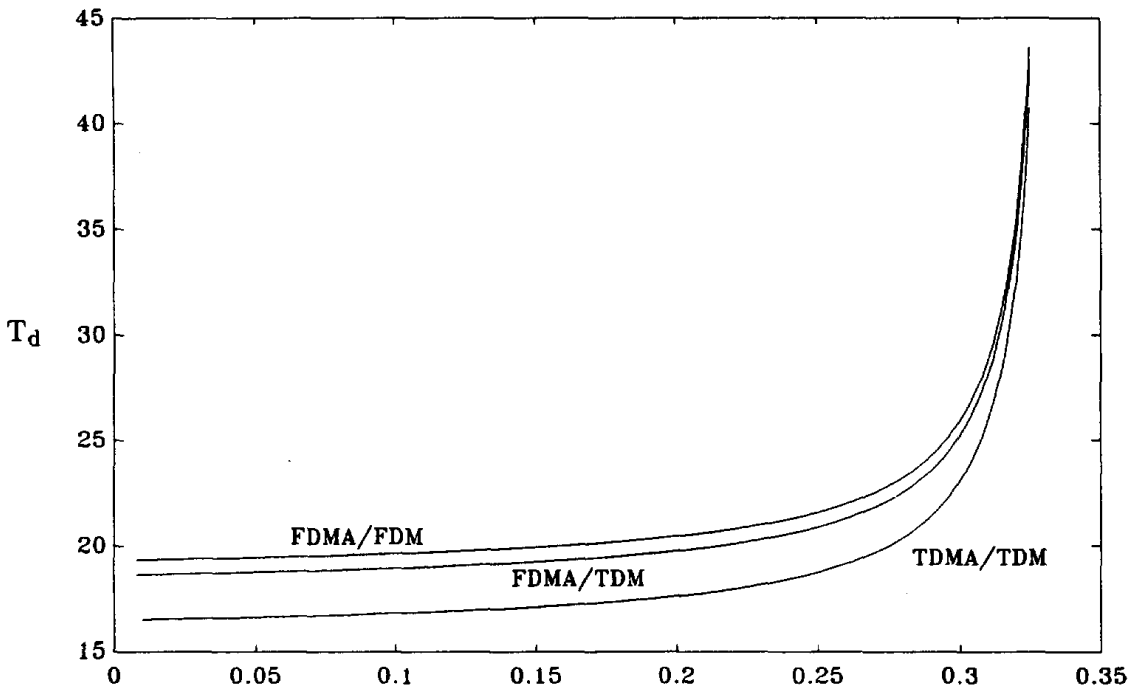

Fig. 3 Comparison among the three protocols for $m=10, n=10$, $\rho=0.8, \mathrm{t}_{\mathrm{b}}=\tau / 20, \mathrm{t}_{0}=3 \tau, \mathrm{t}_{\mathrm{b}^{\prime}}=\mathrm{t}_{2} / 20, \mathrm{t}_{2}=\mathrm{t}_{\mathrm{o}}=10 \mathrm{t}_{\mathrm{o}}{ }^{\prime}$ 